\title{
Cerebral Hypoxia and Ischemia in the Pathogenesis of Dementia After Stroke
}

\author{
J. T. MORONEY, ${ }^{a}$ E. BAGIELLA, ${ }^{b}$ D. W. DESMOND,${ }^{a, c}$ \\ M. C. PAIK, ${ }^{b}$ Y. STERN, ${ }^{a, d, e}$ AND T. K. TATEMICHI ${ }^{f}$ \\ ${ }^{a}$ Neurological Institute, ${ }^{b}$ Division of Biostatistics, and the ${ }^{d}$ Gertrude H. Sergieusky \\ Center, and the ${ }^{\circ}$ Department of Psychiatry, Columbia University College of \\ Physicians \& Surgeons, New York, New York 10032, USA
}

While it has been reported that ischemic stroke significantly increases the risk of delayed dementia, ${ }^{1,2}$ the underlying mechanisms are not well understood. Hypoxic and ischemic (HI) injury resulting from cerebral hypoperfusion due to systemic illness has been proposed as a pathogenic mechanism in certain subgroups of patients. ${ }^{1.3}$ Thus, the aim of this study was to investigate whether cerebral HI injury resulting from certain systemic illnesses (e.g., cardiac arrhythmias, cardiac failure, pneumonia, seizures, sepsis) would be an independent risk factor for the development of incident dementia after ischemic stroke.

\section{METHODS}

We prospectively followed 185 initially nondemented ischemic stroke patients (mean age $=70.3 \pm 7.7$ years) and identified dementia in annual examinations using modified DSM-III-R criteria based on neuropsychological and functional assessments. A more extensive description of our recruitment and examination procedures is available in an earlier report of our methods and baseline findings. ${ }^{4}$ $\mathrm{HI}$ disorders were defined on the basis of previous reports ${ }^{5,6}$ and identified by means of chart review and/or patient examination during hospitalization. We used KaplanMeier analysis to determine the cumulative proportions of subjects surviving free of dementia in the groups of patients with and without $\mathrm{HI}$ disorders and Cox proportional hazards analysis to estimate the relative risk (RR) of incident dementia associated with the occurrence of these disorders.

\section{RESULTS}

As presented graphically in Figure 1, the cumulative proportion ( $\pm S E$ ) of patients surviving free of dementia in the HI event group was $51.7 \% \pm 10.9$ versus $78.2 \% \pm 4.3$ in the group of patients without a history of $\mathrm{HI}$ disorders after a

\footnotetext{
${ }^{c}$ Address for correspondence and reprint requests: Dr. Desmond, Stroke and Aging Research Project, Neurological Institute, 710 West 168th Street, New York, New York 10032. Phone, 212/305-1809; fax, 212/305-1308; e-mail: dwd2@columbia.edu

${ }^{f}$ Dr. Tatemichi died on April 22nd, 1995.
} 


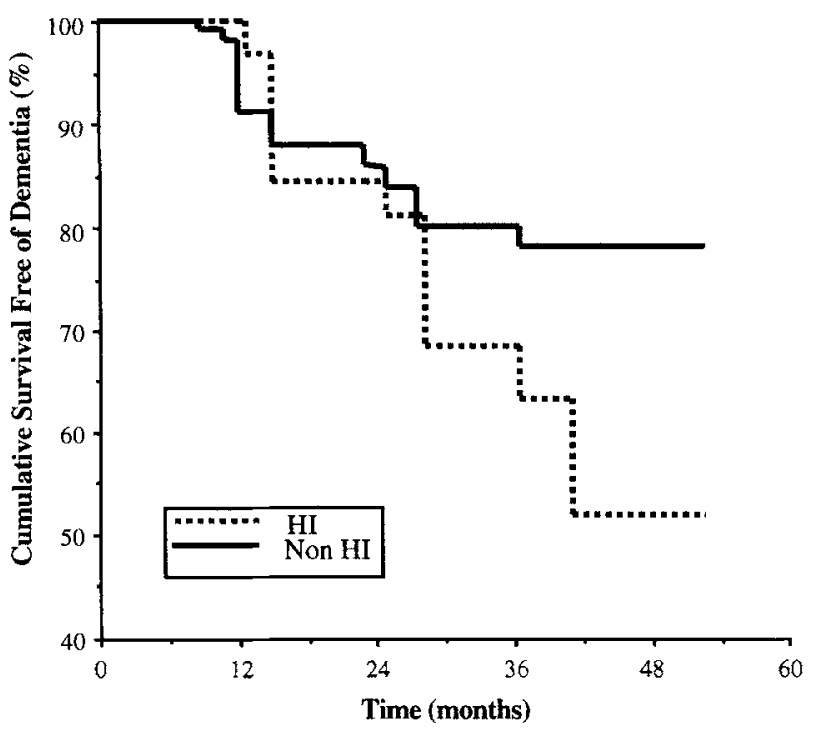

FIGURE 1. Graph of Kaplan-Meier analysis showing the cumulative proportion of patients surviving free of dementia stratified by hypoxic-ischemic $(\mathrm{HI})$ status during the follow-up period up to 53 months.

maximum of 52.8 months of follow-up. As shown in TABLE 1 , the Cox analysis resulted in a relative risk [RR] of 4.28 (95\% confidence interval $[\mathrm{CI}]=1.92$ to 9.55 ) for $\mathrm{HI}$ disorders as a predictor of incident dementia, after adjusting for demographic factors, recurrent stroke, and baseline cognitive function. Age was a significant covariate, with patients aged $70-79$ and $\geq 80$ years being at increased risk $(\mathrm{RR}=$ $2.66,95 \% \mathrm{CI}=1.17$ to 6.06 , and $\mathrm{RR}=3.80,95 \% \mathrm{CI}=1.25$ to 11.60 , respectively) relative to the 60-69-year-old reference group. Baseline MMSE score was also a significant predictor in the final model, with a RR of $3.55(95 \% \mathrm{CI}=1.72$ to 7.33$)$, while recurrent stroke was not.

\section{DISCUSSION}

Our results suggest that $\mathrm{HI}$ disorders may be an independent risk factor for incident dementia after stroke. We found a particularly high risk of delayed dementia in older patients with $\mathrm{HI}$ disorders, suggesting increased vulnerability with advancing age. Although the number of incident dementia cases in the HI patient group was small, our findings support the concept of a "cardiogenic" or hypoperfusion dementia.

Consistent with our findings, Sulkava and Erkinjuntti ${ }^{7}$ have reported that 6 of 133 patients $(4.5 \%)$ with vascular dementia had experienced cerebral hypoperfusion resulting from systemic hypotension due to cardiac arrhythmias. CT brain imaging demonstrated white-matter lesions in five of the six patients, and vascular changes in the deep white matter and atheromatous disease affecting the circle of Willis were noted at autopsy in those patients. The authors speculated that atherosclerosis 
TABLE 1. Relative Risk of Incident Dementia Based on Cox ProportionalHazards Analysis for Hypoxic-Ischemic (HI) Stroke Patients Compared to Non-HI Stroke Patients Adjusting for Demographic Factors, Baseline MiniMental State Examination (MMSE) Score, and Recurrent Stroke As a TimeDependent Covariate

\begin{tabular}{llc}
\hline \multicolumn{1}{c}{ Variable } & \multicolumn{2}{c}{$\begin{array}{c}\text { Relative Risk } \\
\text { (95\% CI) }\end{array}$} \\
\cline { 2 - 3 } & \multicolumn{2}{c}{ Final Model } \\
\hline HI events (yes vs. no) ${ }^{a}$ & 4.28 & $(1.92-9.55)$ \\
Age (vs. 60-69 yr) & & \\
$\quad 70-79$ yr & 2.66 & $(1.17-6.06)$ \\
$\quad 80+$ yr & 3.80 & $(1.25-11.60)$ \\
Education (vs. 13+ yr) & & \\
$\quad \leq 8$ yr & 0.89 & $(0.31-2.57)$ \\
$\quad 9-12$ yr & 1.24 & $(0.39-3.89)$ \\
Sex (women vs. men) & 0.84 & $(0.39-1.80)$ \\
Race (white vs. nonwhite) & 0.47 & $(0.20-1.13)$ \\
Recurrent stroke (yes vs. no) & 1.72 & $(0.72-4.11)$ \\
MMSE (>24 vs. $\leq 24)$ & 3.55 & $(1.72-7.33)$ \\
\hline
\end{tabular}

NOTE. $\mathrm{CI}=$ Confidence Interval; $\mathrm{HI}=$ Hypoxic-ischemic; MMSE $=$ Mini-Mental State Examination at 7-10 or 30 days after stroke (not used for the purpose of dementia diagnosis).

${ }^{\circ}$ Time-dependent covariate.

of the deep penetrating arteries predisposed to hypoperfusion of the white matter in the setting of a decrease in systemic blood pressure. Among 175 consecutive autopsy cases of dementia, Brun ${ }^{3}$ found that $29 \%$ of the patients with vascular dementia had pathologic evidence of cerebral hypoperfusion with either selective incomplete infarction of the white matter or borderzone infarction. In populationbased studies, Skoog et al. ${ }^{8}$ found that $4.1 \%$ of 14785 -year-old patients with dementia had cerebral hypoperfusion as the primary basis for their dementia syndrome, while investigators in the Cardiovascular Health Study ${ }^{9}$ reported that white-matter lesions on brain MRI were significantly associated with episodes of orthostatic hypotension. Finally, misuse of antihypertensive agents can lead to hypotensive episodes and, potentially, to cognitive decline in elderly patients. ${ }^{10}$

In conclusion, cerebral $\mathrm{HI}$ injury resulting from hypoperfusion due to systemic hypotension in the setting of co-morbid medical illnesses or misuse of antihypertensive agents may be an important pathogenic mechanism in the development of incident dementia after stroke. Early recognition of $\mathrm{HI}$ disorders may allow targeted therapeutic interventions to prevent subsequent cognitive deterioration in vulnerable patients.

\section{REFERENCES}

1. TAtemichi, T. K., M. Paik \& E. Bagrella et al. 1994. Risk of dementia after stroke in a hospitalized cohort: Results of a longitudinal study. Neurology 44: 1885-1891.

2. Kokmfn, E., J. P Whisnant, W. M. O'Fallon et al. 1996. Dementia after ischemic stroke: A population-based study in Rochester, Minnesota (1960-1984). Neurology 19: $154-159$.

3. Brun, A. 1994. Pathology and pathophysiology of cerebrovascular dementia: Pure subgroups of obstructive and hypoperfusive etiology. Dementia 5: 145-147. 
4. Tatemichi, T. K., D. W. Desmond, R. Mayeux et al. 1992. Dementia after stroke: Baseline frequency, risks, and clinical features in a hospitalized cohort. Neurology 42: $1185-1193$.

5. Ginsberg, M. D., E. T. Hedley-Whyte \& E. P. Richardson. 1976. Hypoxic-ischemic leukoencephalopathy in man. Arch Neurol 33: 5-14.

6. Brierley, J. B. \& D. I. Graham. 1984. Hypoxia and vascular disorders of the central nervous system. In Greenfield's Neuropathy, 4th ed. J. H. Adams, J. A. N. Corsellis \& L. W. Duchen, Eds.: 125-156. Edward Arnold. London.

7. Sulkava, R. \& T. ERkinjuntti. 1987. Vascular dementia due to cardiac arrhythmias and systemic hypotension. Acta Neurol. Scand. 76: 123-128.

8. Skoog, i., L. Nilsson, B. Palmertz, L. A. Andreasson \& A. Svanborg. 1993. A population-based study of dementia in 85-year-olds. N. Engl. J. Med. 328: 153-158.

9. LoNGSTRETH, W. T., T. A. MANOLIO, A. ARNOLD et al. 1996. Clinical correlates of white matter findings on cranial magnetic resonance imaging of 3301 elderly people: The Cardiovascular Health Study. Stroke 27: 1274-1282.

10. Jansen, P. A. F, F. W. J. Gribnau, B. P. M. Schulte \& E. F. J. Poels. 1986. Contribution of inappropriate treatment for hypertension to pathogenesis of stroke in the elderly. Br. Med. J. 293: 914-917. 